\title{
EXPERIENCIAS DE UNA DÉCADA DE SEGUIMIENTO FARMACOTERAPÉUTICO A PACIENTES DE LA COMUNIDAD EN UNA UNIVERSIDAD PÚBLICA
}

\author{
Experiences of a decade of pharmacotherapeutic follow up to patients from a community at a \\ public university \\ Gladys M Delgado $\mathrm{P}^{1}$, Doris H Delgado $\mathrm{P}^{2}$, María del R Carreño Q ${ }^{1}$, William Cortez $\mathrm{M}^{1}$ \\ ${ }^{1}$ Gabinete de Atención Farmacéutica, Facultad de Farmacia y Bioquímica, Universidad Nacional Mayor de San Marcos \\ ${ }^{2}$ Facultad de Medicina, Universidad Nacional Mayor de San Marcos
}

\section{RESUMEN}

El seguimiento farmacoterapéutico (SFT) es una práctica profesional en la cual el farmacéutico se hace responsable del paciente en relación a su farmacoterapia, mediante intervenciones farmacéuticas al prevenir, identificar y resolver problemas relacionados con los medicamentos (PRM). El objetivo del presente, fue describir las experiencias de una década de SFT a pacientes de la comunidad en una universidad pública. El estudio fue descriptivo observacional. Los datos se obtuvieron de los Cuadernos de registro, Hojas de SFT y Hojas de evaluación de la intervención del Gabinete de Atención Farmacéutica de la Facultad de Farmacia y Bioquímica de la Universidad Nacional Mayor de San Marcos UNMSM; los cuales fueron procesados y evaluados en excel, obteniéndose los siguientes resultados: Las atenciones a los pacientes fueron efectuadas por residentes de Farmacia Clínica, desde octubre del 2003 hasta mayo del 2012. Recibieron intervención farmacéutica 287 pacientes: 163 mujeres y 124 varones; 90 pacientes con SFT ( 35 asistieron menos de un mes, 29 de un mes a 1 año, y 26 más de 1 año). Los PRM encontrados fueron 360 (279 reales: 181 PRM1, 9 PRM2, 11 PRM3, 30 PRM4, 11 PRM5, 37 PRM6; los otros 81 fueron PRM potenciales). En estos 36o PRM se observan: 114 intervenciones farmacéuticas que cumplen objetivos terapéuticos (dosis alternativa (39\%), medicamento incluido (39\%), medicamento alternativo (15\%), nuevo esquema terapéutico (9\%), suspensión de la prescripción (7\%).

Palabras claves: Atención farmacéutica, seguimiento farmacoterapéutico, farmacia comunitaria.

\section{SUMMARY}

The pharmacotherapeutic follow up $(\mathrm{PF})$ is a practice in which the pharmacist is responsible for the patients in relation to their drugs through pharmaceutical interventions to prevent, identify and solve drugs therapy problems (DTP). The aim of this study was to describe the experiences of a decade of PF in patients from the community at a public university. The study was descriptive and observational. The data were obtained from the notebooks, PF sheets and evaluation intervention sheets of Cabinet of Pharmaceutical Care of Faculty of Pharmacy and Biochemistry, San Marcos University; which were processed and evaluated in excel, with the following results: the attention to patients were made by residents of Clinical Pharmacy, from October 2003 to May 2012. 287 patients received pharmaceutical intervention: 163 women and 124 men, 90 patients with PF (35 attended less than a month, 29 a month to 1 year, and 26 over 1 year). The DTP found were 360 (279 reals: 181 DTP1, 9 DTP2, 11 DTP3, 30 DTP4, 11 DTP5, 37 DTP6) and_the other 81 were potential DTPs). In these 360 DTP is observed: 114 pharmaceutical interventions that meet therapeutic goals (alternative dose (39\%), including medicine (39\%), alternative medicine ( $15 \%)$, new therapeutic regimen (9\%), suspension of the prescription (7\%).

Keywords: Pharmaceutical Care, pharmacotherapy follow, community pharmacy.

\section{INTRODUCCIÓN}

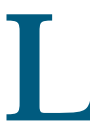

a Atención Farmacéutica en el Perú se va perfeccionando y sistematizando cada vez más, lo que puede evidenciarse con los logros obtenidos en el desarrollo de esta actividad, concretamente en la implementación del SFT, en el manejo de información de medicamentos y en la farmacovigilancia. Actualmente es posible observar que se está desarrollando el conocimiento científico requerido para su aplicación ${ }^{(1,2)}$.
En la práctica, estos logros concretos, se deben a que la Universidad Nacional MayordeSan Marcos, desdela década de los 80 viene formando especialistas en Farmacia Clínica, y desde los 90 inicia la implementación de los Servicios de Atención Farmacéutica en sus sedes de residentado hospitalario en farmacia clínica, donde se desarrolla el SFT a pacientes hospitalizados. En la misma década la universidad inauguró el Gabinete de Atención Farmacéutica para desarrollar el SFT a pacientes de la comunidad ${ }^{(3)}$.

Estas actividades, que han sido oficializadas con dispositivos legales dados a través del Ministerio de 
Salud, son de aplicación obligatoria en los centros de salud del país, de tal manera que en la Política Nacional de Medicamentos, en lo referido a la Promoción del Uso Racional de Medicamentos, se incide en la "información y educación para los usuarios". La Ley del Químico Farmacéutico (4), menciona entre sus funciones "brindar atención farmacéutica en farmacias y boticas del sector público y privado". La Resolución Ministerial $\mathrm{N}^{\circ}$ 552-2007 MINSA ${ }^{(5)}$, hace referencia a la Red Nacional del Sistema de Dispensación de Medicamentos en Dosis Unitaria. El Manual de Buenas Prácticas de Dispensación ${ }^{(6)}$, tiene como objetivos específicos: "Orientar a los pacientes para el uso adecuado de los medicamentos", "Contribuir al SFT de pacientes según criterios específicos" e "Identificar y contribuir a la solución de los problemas relacionados con el uso de los medicamentos". A ellos se suma el Reglamento de Establecimientos Farmacéuticos (7), en el que se pueden observar algunas definiciones importantes para la Atención Farmacéutica.

Esta combinación del soporte legal que emana de la autoridad, con el soporte técnico a través de la formación de especialistas y profesionales de postgrado en las universidades, con metodologías modernas de enseñanza mediante competencias que van a la vanguardia de los acontecimientos, van a determinar que en la práctica se desarrolle una AF con una eficiencia tal que alcance el mejoramiento continuo, para mejorar la calidad de vida del paciente.

El objetivo de este estudio ha sido describir las experiencias de una década de SFT a pacientes de la comunidad en una universidad pública.

\section{MATERIALES Y MÉTODOS}

El enfoque del estudio fue cuantitativo, de tipo observacional, descriptivo, retrospectivo, de corte transversal. Se realizó en el Gabinete de Atención Farmacéutica (GAF), de la Facultad de Farmacia y Bioquímica de la Universidad Nacional Mayor de San Marcos. La población estuvo conformada por los pacientes que asistieron a recibir atención en este GAF; la muestra fueron todos los pacientes que recibieron AF con SFT registrados en las "Hojas de registro de seguimiento farmacoterapéutico" (Hoja de anamnesis farmacológico, Hoja farmacoterapéutica, Hoja de evaluación integral de sospechas, Hoja de evaluación de la intervención farmacéutica) desde octubre 2003 hasta marzo 2012.

Antes de iniciar el SFT en el GAF, se desarrollaron, desdeel año 2002, los procedimientos para suaplicación en pacientes de la comunidad, teniendo como base la experiencia obtenida en pacientes hospitalizados. Esta metodología, denominada "Gestión de casos clínicos", consta de 5 procesos: (1) Selección del paciente, (2) Obtención de datos del paciente, (3) Análisis, evaluación e identificación del PRM, (4) Plan del SFT, (5) Evaluación de la intervención farmacéutica.

Los PRM propuestos se detallan a continuación:

\section{Por necesidad}

Necesita un medicamento que no usa

PRM1

Usa un medicamento que no necesita

$\mathrm{PRM} 2$

\section{Por efectividad}

Medicamento inefectivo, independiente de la dosis

$\mathrm{PRM}_{3}$

Dosis, intervalo o duración inferior a la necesaria

$\mathrm{PRM}_{4}$

\section{Por seguridad}

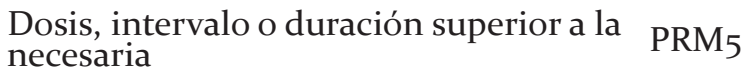

Provoca una reacción adversa al PRM6 medicamento

Las intervenciones farmacéuticas propuestas fueron las siguientes: Alternativas terapéuticas, Sugerir la suspensión de la prescripción propuesta al médico tratante, Modificación de la prescripción en coordinación con el médico, Medicamento alternativo, Dosis alternativa, Forma y vía alternativa, Modificación de la duración de terapia, Disminución del número de fármacos, Medicamento incluido, Prevención de eventos adversos (RAM, Interacción Medicamentosa, Toxicidad, agravamiento de Patología), Educación del paciente.

La implementación de estos procesos en el GAF se inicia desde octubre del 2003, con resultados registrados. Asimismo, cabe mencionar que en el GAF no existe un farmacéutico asignado para esta labor de forma permanente, por lo que las atenciones han sido desarrolladas por los Q.F. de la especialidad en Farmacia Clínica, quienes obligatoriamente realizan una rotación por este servicio, que sólo funciona con la permanencia de ellos.

Para el análisis, los datos se digitaron en hojas de cálculo Excel versión 10. Los resultados y figuras que se muestran se obtuvieron del mismo programa. Se determinaron los PRM reales y potenciales detectados, así como los objetivos logrados y las prevenciones realizadas con las intervenciones farmacéuticas al desarrollar el SFT a pacientes que asistieron al GAF, durante el período 2003-2012. 


\section{RESULTADOS}

En el período de estudio se ha obtenido los siguientes resultados:

Las atenciones realizadas a los pacientes de la comunidad en el GAF desde octubre del 2003 a mayo del 2012 se desarrollaron durante el período de permanencia de los QF residentes de Farmacia Clínica, totalizando 56 meses de permanencia física. En ese tiempo se realizaron 2001 atenciones a pacientes comunitarios, 807 con intervención farmacéutica, de las cuales 610 fueron intervenciones farmacéuticas que correspondieron a SFT. (figura 1).

El número de pacientes que recibieron la intervención farmacéutica fueron 287 (163 mujeres y 124 varones), de los cuales 90 fueron los pacientes con SFT y que concurrieron al GAF como se describe: 35 asistieron menos de un mes, 29 asistieron de un mes a 1 año, y 26 más de 1 año (figura 2). Asimismo, de estos 90 pacientes, $84 \%$ visitó de 2 a 4 veces el GAF, $7 \%$ realizó de 7 a 11 visitas, $4 \%$ asistió de 12 a 16 visitas, y 5\% de pacientes realizó más de 16 visitas durante el período de estudio (figura 3 ).

De 36o PRM encontrados, 279 fueron PRM reales, problemas que el farmacéutico observó en el paciente al momento de la entrevista y que fueron clasificados de la siguiente manera: 181 PRM1 (necesitaba un medicamento que no estaba usando), 9 PRM2 (utilizaba un medicamento que no necesitaba), 11 $\mathrm{PRM}_{3}$ (medicamento inefectivo, independiente de la dosis), $30 \mathrm{PRM}_{4}$ (la dosis, el intervalo o la duración fue inferior a la necesaria), $11 \mathrm{PRM}_{5}$ (la dosis, el intervalo o la duración fueron superior a la necesaria), 37 PRM6 (provocó una reacción adversa al medicamento) y 81 fueron PRM potenciales, problemas que el paciente no presentaba al momento de la entrevista, sin embargo de continuar esa situación podría manifestarlos dada las características del paciente, por tanto fueron clasificados según el PRM al que corresponderían: 68 PRM1, 3 PRM2, 3 PRM3, 5 PRM4, 1 PRM5, 1 PRM6 (figura 4 ).

De las 360 intervenciones farmacéuticas, 156 fueron aceptadas por el paciente con cambios de acuerdo a lo sugerido por el farmacéutico, 2 no fueron aceptadas, 178 no tuvieron resultados y 24 no fueron reportadas.

\section{DISCUSIÓN}

El seguimiento farmacoterapéutico es un acto profesional donde el farmacéutico orienta y supervisa al paciente en relación al cumplimiento de su farmacoterapia, mediante intervenciones farmacéuticas, dirigidas a prevenir, identificary resolver problemas relacionados con los medicamentos (PRM).

La importancia de este trabajo está estrechamente ligada a la seguridad del paciente, pretende resolver problemas y prevenir errores. Baena ${ }^{(8)}$ manifiesta que el aporte del farmacéutico a la seguridad del paciente se realiza con la Atención Farmacéutica y con el Seguimiento Farmacoterapéutico (SFT) que previene, detecta y resuelve PRM. El mayor porcentaje de pacientes que aceptaron se les realice SFT estuvo entre

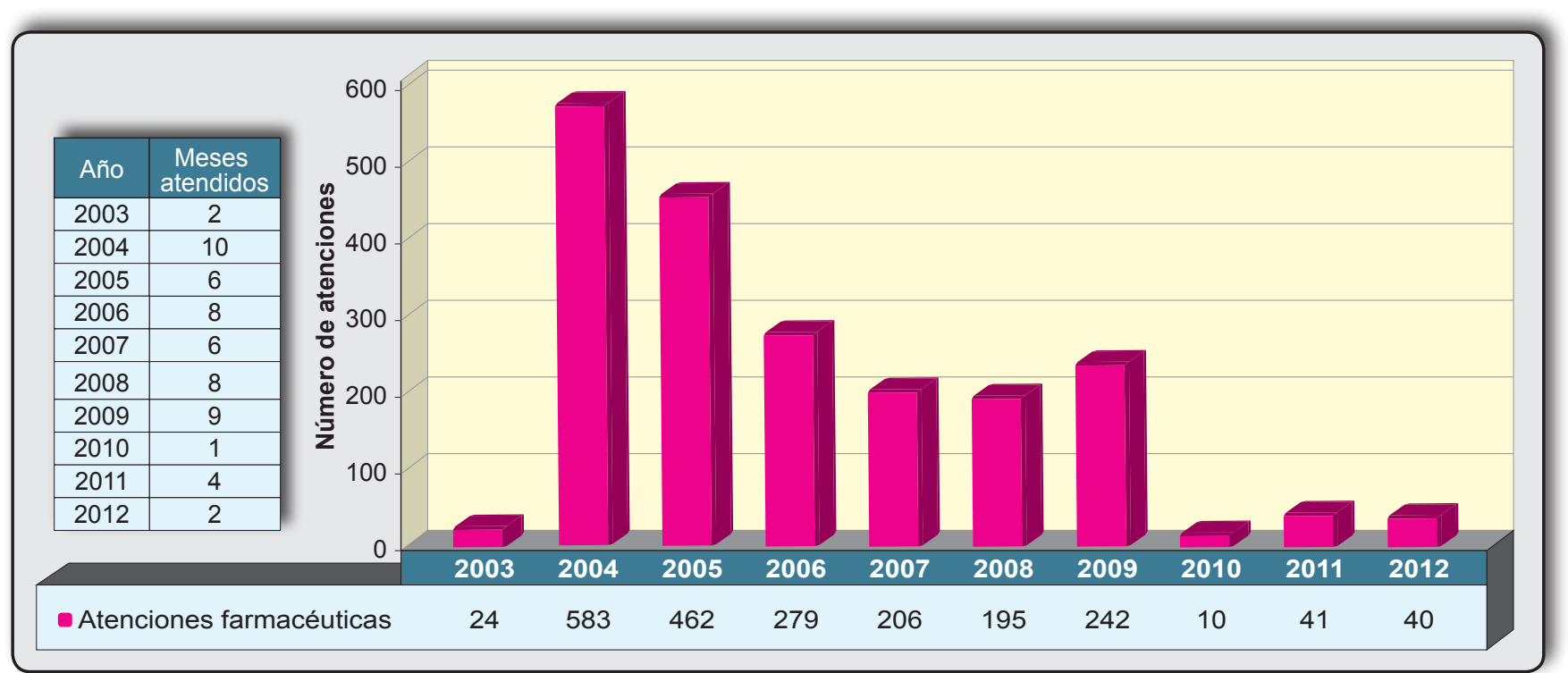

Figura 1. Número de atenciones en el GAF-UNMSM 2003-2012. 
las edades de 63 a 70 años, por lo que debe considerarse que el estudio de Doggrell ${ }^{(9)}$ indica que los pacientes

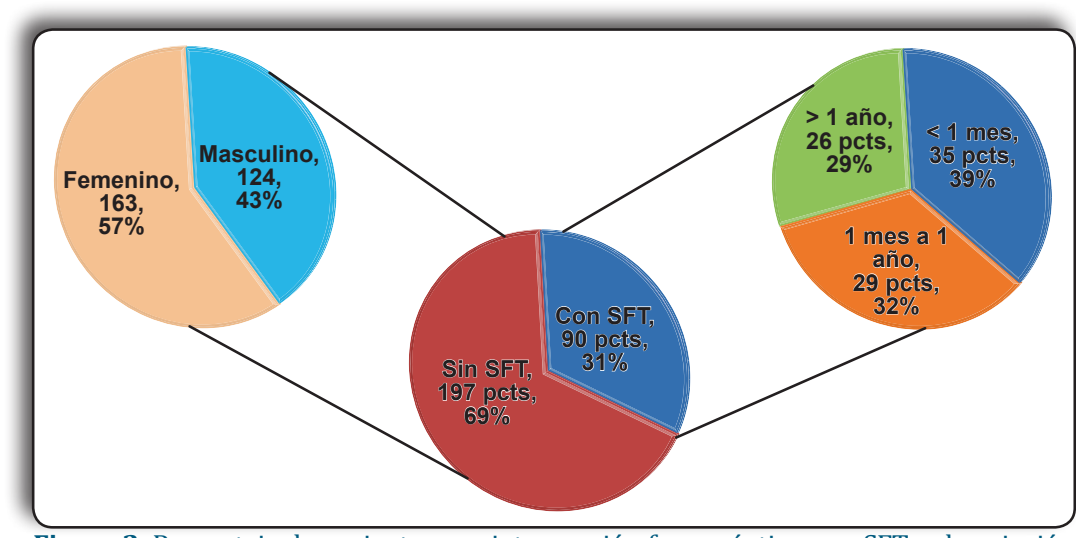

Figura 2. Porcentaje de pacientes con intervención farmacéutica, con SFT y descripción de período de asistencia al GAF-UNMSM 2003-2010.

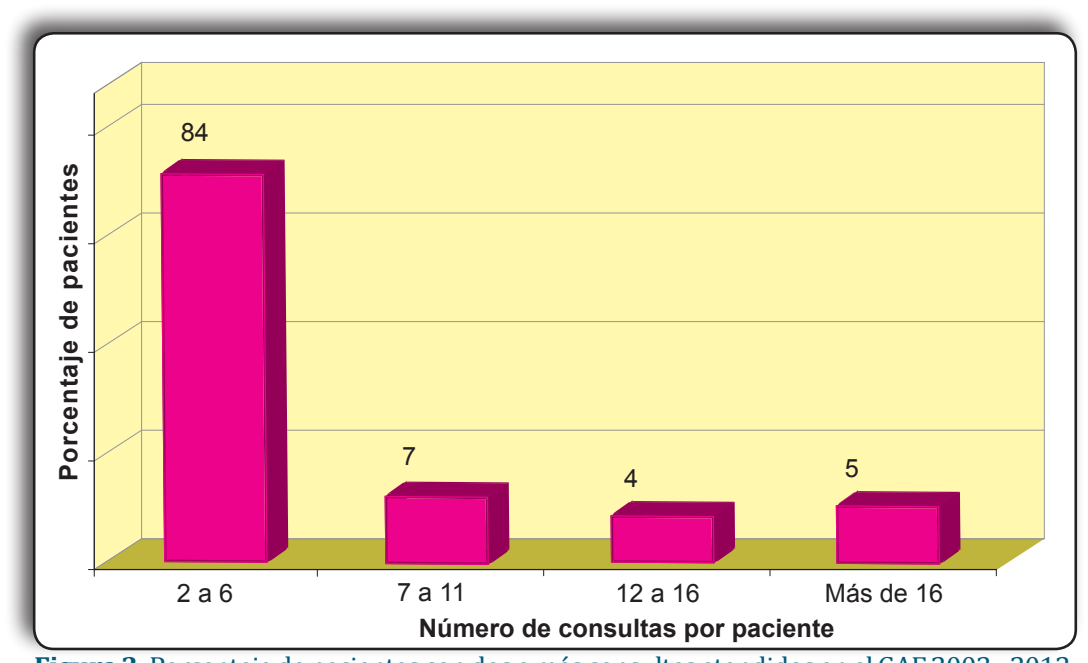

Figura 3. Porcentaje de pacientes con dos a más consultas atendidos en el GAF 2003 - 2012 mayores de 65 años "tienen como aliado" al profesional farmacéutico, observando que con su ayuda se incrementa el grado de adherencia al tratamiento.

El mayor porcentaje de intervenciones farmacéuticas fueron observadas con resultados de Dosis alternativa (39\%) y de Medicamento incluido (39\%), mientras que Medicamento alternativo fue (15\%), seguida de Nuevo esquema terapéutico (9\%). En la Guía para la gestión del uso de medicamentos, elaborada por MINSALChile en 2010, se observan propuestas de indicadores para la evaluación y seguimiento del uso racional de los medicamentos a la Intervención farmacéutica ${ }^{(10)}$.

Iyer, quien trabajó con un grupo de 544 pacientes diabéticos tipo 2 ambulatorios a quienes se logró reducir la HbAic, siendo atendidos por un grupo de especialistas en Farmacia Clínica, señala que una de las principales intervenciones farmacéuticas realizadas fue la educación al paciente ${ }^{(11)}$. Jameson coincide en lo mismo al indicar que la intervención farmacéutica con profesionales capacitados, como se desarrolla en el GAF, permite una mejor intervención ${ }^{(12)}$.

Los farmacéuticos comunitarios acreditados facilitarán la mejora

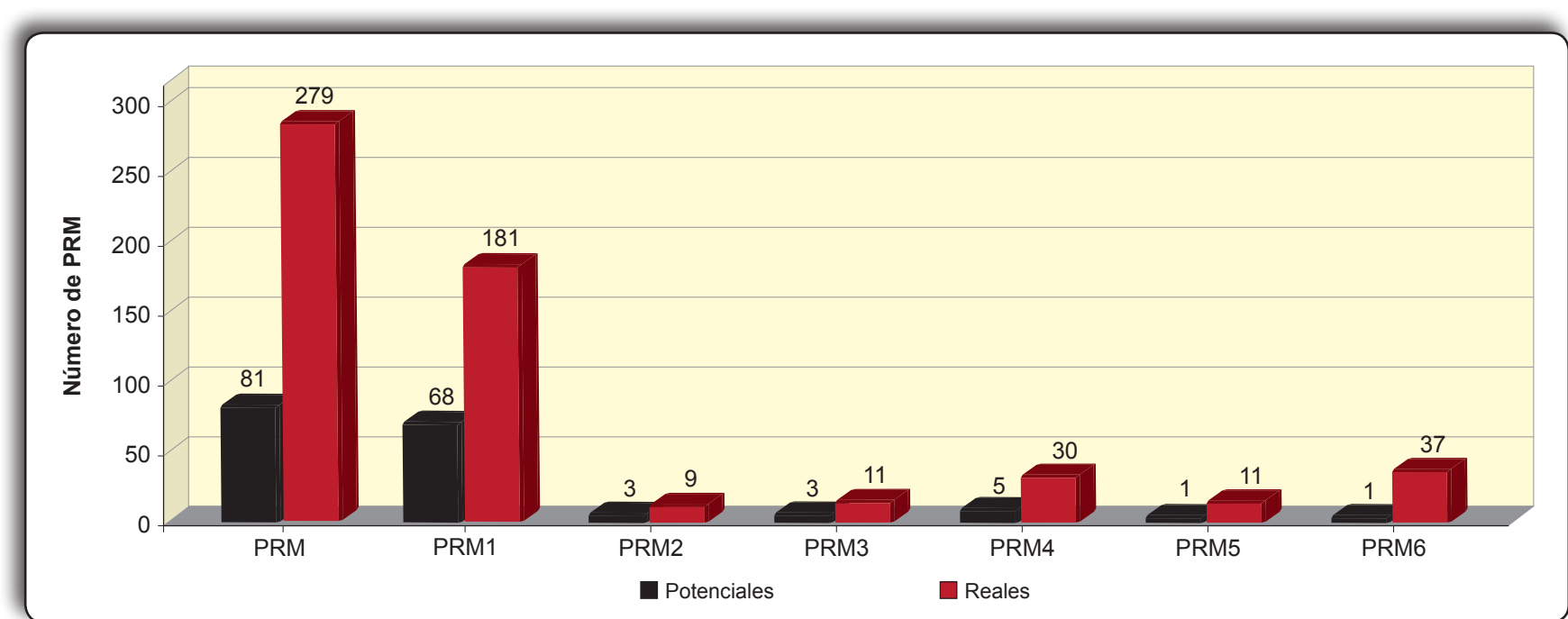

Figura 4. Número de PRM reales y potenciales observados en el GAF-UNMSM 2003-2010. 
del estilo de vida del paciente, la adherencia a los tratamientos y el manejo de sus medicamentos. Los resultados derivados de ello, según el caso particular de cada paciente, podrían ser el cambio del riesgo cardiovascular global, evaluado a través de la ecuación de riesgo de Framingham ${ }^{(13-15)}$, la disminución de la HbAic_(16), o el control de la presión arterial ${ }^{117-}$ ${ }^{18)}$. En todos los casos se resalta la importancia del cuidado primario de estas patologías e indica que los datos clínicos objetivos de los pacientes son buenos indicadores para evaluar el resultado del grupo que se monitorea.

\section{CONCLUSIONES}

1. Durante el funcionamiento del GAF, 287 pacientes recibieron intervención farmacéutica, de los cuales, a 90 se les realizó SFT).

2. Los PRM encontrados fueron 360 (279 reales: siendo 181 PRM1 el de mayor número; los otros 81 fueron PRM potenciales).

3. En los 360 PRM se observan 114 intervenciones farmacéuticas que cumplen objetivos terapéuticos, siendo los de mayor porcentaje Dosis alternativa (39\%) y Medicamento incluido (39\%).

4. Las 224 intervenciones farmacéuticas fueron de prevención (9 de RAM, 216 de patología). Las intervenciones farmacéuticas aceptadas por el paciente fueron 156 .

\section{REFERENCIAS BIBLIOGRÁFICAS}

1. Cipolle RJ, Strand LM, Morley PC. Pharmaceutical Care Practice. $2^{\text {a }}$ ed. McGraw-Hill's. New York, 2008.

2. Provers JP, Currie JD, Phagel HP, McDonough RP, Sobodka JL. A practical guide to pharmaceutical care. $2^{\text {a }}$ ed. APhA. Washington DC, 2003.

3. Delgado PG, Carreño QR, Barreto RL, Hernández PM. Atención Farmacéutica. $1^{\mathrm{a}}$ ed. Lima, 2004.

4. Ley del trabajo del Químico Farmacéutico del Perú. Ley № 28173. (Feb 17, 2004)

5. Norma Técnica de Salud: Sistema de dispensación de medicamentos en dosis unitaria para los establecimientos del sector salud. RM 552-2007/MINSA. (Jul 04, 2007).

6. Manual de Buenas Prácticas de Dispensación. RM o132009/MINSA. (Ene 15, 2009).

7. Reglamento de Establecimientos Farmacéuticos. DS № 014-2011-SA. (Jul 27, 2011).

8. Baena MI, Martínez-Olmos J, Faus MJ, Fajardo P, Martínez-Martínez F. El seguimiento farmacoterapéutico: un componente de la calidad en la atención al paciente. Ars Pharm 2005; 46 (3):213-32.
9. Doggrell SA. Adherence to medicines in the older-aged with chronic conditions: does intervention by an allied health professional help. Drugs Aging 2010; 27 (3): 23954 .

10. Guía para la gestión del uso de medicamentos. Propuestas de indicadores para la evaluación y seguimiento del uso racional de los medicamentos. Ministerio de Salud. República de Chile (Set-2010).

11. Iyer R, Coderre P, McKelvey T, Cooper J, Berger J, Moore E, Kushner M. An employer-based, pharmacist intervention model for patients with type 2 diabetes. Am J Health Syst Pharm. 2010; 67(4): 312-6.

12. Jameson JP, Baty PJ. Pharmacist Collaborative Management of Poorly Controlled Diabetes Mellitus: A Randomized Controlled Trial. Am J Manag Care 2010; 16(4):250-5.

13. Kevin P, Mc Namara, George J, L O’Reilly S, Jackson SL, et al. Engaging community pharmacists in the primary prevention of cardiovascular disease: Protocol for the Pharmacist Assessment of Adherence, Riskand Treatment in Cardiovascular Disease (PAARTCVD). BMC Health Services Research 2010; 10: 264.

14. Al Mazroui NR, Kamal MM, Ghabash NM, Yacout TA, Kole TL, McElnay JC. Influence of pharmaceutical care on health outcomes in patients with Type 2 diabetes mellitus. Br J Clin Pharmacol. 2009; 67(5): 547-57.

15. Johnson CL, Nicholas A, Divine H, Perrier DJ, Blumenschein K, Steinke DT. Outcomes from Diabetes CARE: A pharmacist-provided diabetes management service. J Am Pharm Assoc (2003) 2008; 48(6): 722-30.

16. Mehuys E, Van Bortel L, De Bolle L, Van Tongelen I, Annemans L, Remon P, et al. Effectiveness of a community pharmacist intervention in diabetes care: a randomized controlled trial. J Clin Pharm Ther 2010; 36(5): 602-13.

17. Carter BL, Bergus GR, Dawson JD, Farris KB, Doucette WR, Chrischilles EA, et al. A Cluster Randomized Trial to Evaluate Physician/Pharmacist Collaboration to Improve Blood Pressure Control. J Clin Hypertens (Greenwich) 2008; $10(4): 260-71$.

18. Lau R, Stewart K, McNamara KP, Jackson SH, Hughes JD, Peterson GM, et al. Evaluation of a community pharmacy-based intervention for improving patient adherence to antihypertensives: a randomised controlled trial. BMC Health Serv Res 2010; 10: 34.

Manuscrito recibido el: 18/12/2012

Aceptado para su publicación el: 01/04/2013

\section{Correspondencia:}

Nombre: Gladys Martha Delgado Pérez

Dirección: Jr. Puno 1002 Lima 1-Perú

e-mail: gdelgado@unmsm.edu.pe 